\title{
ON THE COHOMOLOGY OF REAL GRASSMANIANS BY
}

\author{
HOWARD L. HILLER
}

\begin{abstract}
Let $G_{k}\left(\mathbf{R}^{n+k}\right)$ denote the grassman manifold of $k$-planes in real $(n+$ $k)$-space and $w_{1} \in H^{1}\left(G_{k}\left(R^{n+k}\right) ; Z_{2}\right)$ the first Stiefel-Whitney class of the universal bundle. Using Schubert calculus techniques and the cohomology of flag manifolds we estimate the height of $w_{1}$ in the cohomology ring. We then apply this to improve earlier lower bounds on the Lusternik-Schnirelmann category of real grassmanians.
\end{abstract}

The mod 2 cohomology algebra of the grassmanian $G_{k}\left(\mathbf{R}^{n+k}\right)$ of $k$-planes in real $(n+k)$-space processes an interesting duality. By this, we mean that one has at hand two radically different descriptions of the algebraic structure of this cohomology ring. The first one dates back to the enumerative geometry of Schubert [11] and was only much later cast in a topological form by Chern [5]. This approach provides one with an explicit hold of a geometric cell-decomposition of $G_{k}\left(\mathbf{R}^{n+k}\right)$ which induces $Z_{2}$-free generators for the cohomology. On the other hand, one has the computations that arose out of Borel's thesis [4] in terms of the Stiefel-Whitney classes of the universal $k$-plane bundle over $G_{k}\left(\mathbf{R}^{n+k}\right)$. The interplay between these points of view is a substantial and interesting area of research (see [1]). In this paper, we look at the more pedestrian question of estimating the height of the first Stiefel-Whitney class $w_{1}$. This work then finds an immediate topological application to finding lower bounds for the Lusternik-Schnirelmann category of $G_{k}\left(\mathbf{R}^{n+k}\right)$. Elsewhere, we will use similar techniques to discuss divisibility properties of the degrees of certain irreducible representations of the symmetric groups. We now provide a more detailed description of the contents of this paper.

In $\S 1$ we briefly review the descriptions of $H^{*}\left(G_{k}\left(\mathbf{R}^{n+k}\right) ; \mathbf{Z}_{2}\right)$ due to Borel and Chern mentioned above. In particular, we record the classical formulae of Pieri and Giambelli.

In $\$ 2$ we give a completely algebraic method of finding upper bounds on the height of $w_{1}$ using well-known facts about the complete flag manifold and its cohomology. As an easy corollary, we retrieve a result of Berstein [2] on the cup-length of $G_{k}\left(\mathbf{R}^{n+k}\right)$ avoiding his combinatorial analysis of a certain generating function.

In $\$ 3$ we use the full power of the Schubert calculus to find lower bounds on the height of $w_{1}$ that are very close to the upper bounds obtained in $\S 2$. This gives us a fairly good picture of how the height of $w_{1}$ varies as the dimension of the ambient space increases.

Received by the editors September 1, 1978.

AMS (MOS) subject classifications (1970). Primary 57F15; Secondary 55C30. 
Finally, in $\$ 4$ we discuss Lusternik-Schnirelmann category and do a little more work involving $w_{2}$ to obtain better results for the category of grassman manifolds of 3-planes.

In an Appendix, we show how to contract Borel's ideal in the $w_{i}$ and $\bar{w}_{i}$ back to the $w_{i}$ alone. This is described by a fairly simple recursion and is useful in the analysis of $H^{*}\left(G_{2}\left(\mathbf{R}^{n+2}\right) ; \mathbf{Z}_{2}\right)$. The arguments are completely algebraic and elementary.

Some of the results obtained here were motivated by computations done on the MACSYMA system at the Laboratory for Computer Science, MIT. It is a pleasure to thank David Spear and David Barton for their helpful programming. The author would also like to thank Israel Berstein for listening to his original arguments and making helpful suggestions.

1. Preliminaries. We begin by recalling that $G_{k}\left(\mathbf{R}^{n+k}\right)$ is a compact, smooth manifold of dimension $n k$. Let $\zeta_{k, n}$ denote the universal $k$-plane bundle over $G_{k}\left(\mathbf{R}^{n+k}\right)$. The total space consists of pairs $(V, x)$ where $V \subseteq \mathbf{R}^{n+k}$ is a $k$-plane with $x \in V$; and the bundle map is a projection on the first factor. We denote by $w_{i}$ (respectively $\bar{w}_{j}$ ) the $i$ th (respectively $j$ th normal) Stiefel-Whitney class of $\zeta_{k, n}$, $1<i<k, 1<j \leqslant n$. One can view the normal Stiefel-Whitney class $\bar{w}_{j}$ as the Stiefel-Whitney class of the $n$-plane bundle $\bar{\zeta}_{k, n}$ complementary to $\zeta_{k, n}$, given explicitly by the projection $(W, x) \mapsto W^{\perp}$ with $x \in W^{\perp}$. It is clear that

$$
w\left(\zeta_{k, n}\right) w\left(\bar{\zeta}_{k, n}\right)=1
$$

where $w($ ) denotes the total Stiefel-Whitney class, and indeed this is the only relation.

THEOREM 1.1 (BOREL). (i) If $I_{k, n}$ denotes the ideal generated by (1.0) then

$$
H^{*}\left(G_{k}\left(\mathbf{R}^{n+k}\right) ; \mathbf{Z}_{2}\right) \approx \mathbf{Z}_{2}\left[w_{1}, \ldots, w_{k}, \bar{w}_{1}, \ldots, \bar{w}_{n}\right] / I_{k, n} .
$$

(ii) In addition, if $i: G_{k}\left(\mathbf{R}^{n+k}\right) \rightarrow G_{k}\left(\mathbf{R}^{m+k}\right)$ is the inclusion map $(m>n)$ then $i^{*}\left(w_{i}\right)=w_{i}$.

The final statement (ii) is a consequence of the existence of an obvious lifting of the inclusion map to a bundle map, which then respects the Stiefel-Whitney classes. We will often have occasion to exploit this result.

Deciding whether a polynomial in the $w_{i}$ is zero inside this algebra can of ten be a nontrivial task. We will show how to simplify many of these computations by a result of the Appendix. More classically, one can use the topological Schubert techniques of Chern. Chern [5] exploits the cellular decomposition of Ehresmann [6] to identify an additive free basis for the cohomology and then describes the multiplication table for these generators. We briefly describe this construction for the grassman manifold $G_{k}\left(\mathbf{R}^{n+k}\right)$. A Schubert symbol is a sequence $\left[a_{1}, \ldots, a_{k}\right]$ of integers satisfying $0 \leqslant a_{1} \leqslant a_{2} \leqslant \cdots \leqslant a_{k} \leqslant n$. One defines the corresponding Schubert cell

$$
X\left[a_{1}, \ldots, a_{k}\right]=\left[X \in G_{k}\left(\mathbf{R}^{n+k}\right): \operatorname{dim}\left(X \cap \mathbf{R}^{i+a_{1}}\right)>i\right]
$$


where $X\left[a_{1}, \ldots, a_{k}\right]$ is a cell of dimension $a_{1}+\cdots+a_{k}$ and induces a nontrivial mod 2 cycle. Denote by $\left(a_{1}, \ldots, a_{k}\right)$ the dual cocycle. We recall that the StiefelWhitney classes are themselves Schubert cocycles (cycles, for short), i.e.

$$
\bar{w}_{j}=(0, \ldots, 0, j) \text { and } w_{i}=(0, \ldots, 0, \underbrace{1, \ldots, 1}_{i})
$$

where $1 \leqslant i \leqslant k, 1 \leqslant j \leqslant n$. One often refers to the $\bar{w}_{j}$ as special Schubert cycles. They are special, from one point of view, since there is a combinatorial formula describing the multiplication of a special Schubert cell by an arbitrary one, namely $\left(a_{1}, \ldots, a_{k}\right) \bar{w}_{j}=\Sigma\left(b_{1}, \ldots, b_{k}\right)$ where the sum ranges over $k$-tuples $\left(b_{1}, \ldots, b_{k}\right)$ such that $a_{i} \leqslant b_{i} \leqslant a_{i+1}, 1 \leqslant i \leqslant k$ (where $a_{k+1}=n$ ) and $b_{1}+\cdots+b_{k}=j+a_{1}$ $+\cdots+a_{k}$. The formula is often called Pieri's formula and is related to the multiplication of Schur functions [14]. It has as a consequence the classical formula of Giambelli expressing an arbitrary Schubert cycle as a polynomial in the special ones $\left(a_{1}, \ldots, a_{k}\right)=\operatorname{det}\left(\bar{w}_{a_{i}+i-j}\right)$ where $\bar{w}_{0}=1$ and $\bar{w}_{j}=0$ for $j<0$ or $j>n$. For example, an easy consequence of this machinery is a particularly simple description of the fundamental class of the grassmanian $(n, \ldots, n)$.

LEMMA 1.2. The following identity holds in $H^{*}\left(G_{k}\left(\mathbf{R}^{n+k}\right) ; \mathbf{Z}_{2}\right), w_{k}^{j}=(j, \ldots, j)$.

Proof. Induct on $j$.

The Schubert cycle description of the cohomology we have given actually is a much more pervasive phenomenon than we have indicated. Indeed, if $G$ is a complex semisimple Lie group and $P$ is a parabolic subgroup of $G$ then the integral cohomology of $G / P$ admits an analogous free Schubert basis via the Bruhat decomposition. Our exposition only concerns $G=\mathrm{GL}_{n}$ with $P$ a maximal parabolic. We hope to investigate the general situation in a future paper.

2. Flag manifolds and Berstein's theorem. We begin by recalling a familiar notion from algebraic topology. Given a topological space $X$, we define the $(\bmod 2)$ cup-length of $X, \operatorname{cup}(X)$, to be the length of a longest nonzero monomial in the $\mathbf{Z}_{2}$-algebra $H^{*}\left(X ; \mathbf{Z}_{2}\right)$, i.e. if $x_{1} \cup \cdots \cup x_{n} \neq 0$, then $\operatorname{cup}(X)>n$. Berstein [2] considered some aspects of the computation of $\operatorname{cup}\left(G_{k}\left(\mathbf{R}^{n+k}\right)\right)$. He showed

$$
\left.\operatorname{cup}\left(G_{k}\left(\mathbf{R}^{n+k}\right)\right)<n k \quad \text { unless } k \leqslant 2 \text { (where, if } k=2 \text { then } n+1 \text { is a power of } 2\right) \text {. }
$$

His argument employed the Schubert calculus and an ad hoc combinatorial argument. Our proof in the following sections avoids this detail and actually gives more information on the height of $w_{1}$.

We first consider the complete flag manifold $F \operatorname{lag}\left(\mathbf{R}^{m}\right)$ whose points are flags $0<V_{1}<V_{2}<\cdots<V_{m-1}<\mathbf{R}^{m}$ where the dimension of $V_{i}$ is $i$. This is a compact, smooth manifold of dimension $m(m-1) / 2$. There exist $m$ distinct line bundles on Flag $\left(\mathbf{R}^{m}\right)$ which yield one-dimensional classes $e_{1}, \ldots, e_{m}$. These generate the cohomology (see [8])

$$
H^{*}\left(\operatorname{Flag}\left(\mathbf{R}^{m}\right) ; \mathbf{Z}_{2}\right) \approx \mathbf{Z}_{2}\left[e_{1}, \ldots, e_{m}\right] / S_{m}
$$


where $S_{m}$ is the ideal generated by the elementary symmetric functions $\sigma_{j}\left(e_{1}, \ldots, e_{m}\right), 1 \leqslant j \leqslant m$. It is well known that the projection map

$$
\rho: \operatorname{Flag}\left(\mathbf{R}^{n+k}\right) \rightarrow G_{k}\left(\mathbf{R}^{n+k}\right)
$$

defined by sending $0<V_{1}<\cdots<V_{n+k-1}<\mathbf{R}^{n+k}$ to $V_{k}$ induces an injection on cohomology. Indeed, the map $\rho^{*}$ can be described by

$$
\rho^{*}\left(w_{i}\right)=\sigma_{i}\left(e_{1}, \ldots, e_{k}\right) \text { and } \rho^{*}\left(\bar{w}_{j}\right)=\sigma_{j}\left(e_{k+1}, \ldots, e_{k+n}\right)
$$

where $1 \leqslant i \leqslant k, 1 \leqslant j \leqslant n$. We now obtain our first estimate.

Proposition 2.2. In $H^{*}\left(G_{k}\left(\mathbf{R}^{2^{s}}\right) ; \mathbf{Z}_{2}\right), w_{1}^{2^{s}}=0$.

Proof. Since $\rho^{*}$ is an injection, it suffices to show

$$
0=\rho^{*}\left(w_{1}^{2^{s}}\right)=\rho^{*}\left(w_{1}\right)^{2^{s}}=\sigma_{1}\left(e_{1}, \ldots, e_{k}\right)^{2^{s}}=e_{1}^{2^{s}}+\cdots+e_{k}^{2^{s}} .
$$

Certainly, $e_{i}$ is a root of the polynomial

$$
\prod_{i=1}^{2^{s}}\left(X-e_{i}\right)=X^{2^{s}}-\sigma_{1} X^{2^{s}-1}+\cdots+\sigma_{2^{s}}
$$

so we get $e_{i}^{2^{s}}=\sum_{i=1}^{2^{s}}(-1)^{i+1} \sigma_{i} X^{2^{s}-1}$. Thus $e_{1}^{2^{s}}+\cdots+e_{k}^{2^{s}}$ is in the ideal $S_{2^{s}}$ generated by the elementary symmetric functions in $e_{1}, \ldots, e_{2}$ and the proof is complete.

We now get

THEOREM 2.3. If $k>3$ or $k=2, n \neq 2^{s}-1$ then in $H^{*}\left(G_{k}\left(\mathbf{R}^{n+k}\right) ; \mathbf{Z}_{2}\right), w_{1}^{k n}=0$.

Proof. Suppose $k>3$. We write $n=\left(2^{s}-k\right)+j, 0<j<2^{s}$. Inside the cohomology of $G_{k}\left(\mathbf{R}^{2^{s+1}}\right)$ we have, by Proposition 2.2 , that $w_{1}^{2^{s+1}}=0$. The cohomology restriction map

$$
i^{*}: H^{*}\left(G_{k}\left(\mathbf{R}^{2^{s+1}}\right) ; \mathbf{Z}_{2}\right) \rightarrow H^{*}\left(G_{k}\left(\mathbf{R}^{n+k}\right) ; \mathbf{Z}_{2}\right)
$$

preserves the Stiefel-Whitney classes, so it suffices to check the inequality $2^{s+1}<$ $k\left(2^{s}-k+j\right)$. Without loss of generality we can also assume $j=1$. The inequality then becomes $k(k-1)<(k-2) 2^{s}$. But, by symmetry considerations we can assume $k<$ codimension $=2^{s}-k+1$, i.e. $2 k<2^{s}+1$. Of course, parity considerations actually yield $2 k \leqslant 2^{s}$ or $k<2^{s-1}$. Now the inequality becomes $(k-1)<2(k-2)$ which is easily seen to be equivalent to our initial assumption that $k>3$. A precisely analogous argument yields the same result if $k=2$ and $j>1$.

It is now quite easy to obtain one direction of Berstein's result (2.0).

COROLlaRY 2.4. If $k$ and $n$ are as in Theorem 2.3 then $\operatorname{cup}\left(G_{k}\left(\mathbf{R}^{n+k}\right)\right)<k n$.

Proof. This is clear since the cup length can equal the dimension only if the powers of a one-dimensional class survive to the top dimension and this is ruled out by Theorem 2.3.

We can also extract slightly more informaton out of the proof of Theorem 2.3 that we record for later reference. Recall that the height of a cohomology class $z$ is the unique $m$ such that $z^{m} \neq 0$ but $z^{m+1}=0$. 
Corollary 2.5. If $n=\left(2^{s+1}-k\right)-j, 0<j<2^{s}$, then the height of $w_{1}$ in $G_{k}\left(\mathbf{R}^{n+k}\right)$ is $<2^{s+1}$.

Proof. We simply apply Theorem 2.3 and the fact that, for fixed $k$, as the codimension $n$ increases so does the height of $w_{1}$. This follows immediately from Theorem 1.1(ii).

In order to complete the proof of (2.0), we must check that $w_{1}$ survives to the top dimension in the "exceptional" cases (i.e. those not covered by Theorem 2.3). Of course, we need only consider the case $k=2$, since $k=1$ yields the familiar projective space $\mathbf{R P}^{n}$, whose cohomology is a truncated polynomial algebra on $w_{1}$. To do this we use some results of the next section.

Proposition 2.6. In $H^{*}\left(G_{2}\left(\mathbf{R}^{2^{s}+1}\right) ; \mathbf{Z}_{2}\right), w_{1}^{2^{s+1}-2} \neq 0$.

Proof. By Propositions 3.1 and $3.2, w_{i}^{2^{s}}=\left(1,2^{s}-1\right)$. Since $2^{s}-1$ is the codimension, the Pieri formula yields $w_{1}^{2^{s}+i}=\left(1+i, 2^{s}-1\right), i>0$. In particular, $w_{1}^{2^{++1}-2}=$ $\left(2^{s}-1,2^{s}-1\right) \neq 0$.

This result has apparently been known for many years. We give an alternative algebraic proof, without the Schubert calculus, in the Appendix.

3. Lower bounds on the height of $w_{1}$. We resort to the Giambelli formula as described in $\$ 1$ to identify lower bounds on the height of $w_{1}$. Our approach is similar to that of $\$ 2$ in that we compute the exact height of $w_{1}$ along a cofinal sequence of codimensions using the flag manifolds. Here the important dimensions for the ambient space are those of the form $2^{s}+1$ rather than $2^{s}$. We first use the Schubert technique to prove

Proposition 3.1. In $H^{*}\left(G_{k}\left(\mathbf{R}^{2^{s+1}}\right) ; \mathbf{Z}_{2}\right)$,

$$
\bar{w}_{2^{s}+1-k} W_{k-1}=\left(1,1, \ldots, 1,2^{s}-k+1\right) .
$$

Proof. We can write

$$
\left(1, \ldots, 1,2^{s}-k+1\right)=\operatorname{det}\left|\begin{array}{ccccc}
\bar{w}_{1} & 1 & & & \\
\bar{w}_{2} & \bar{w}_{1} & 1 & \\
\vdots & & \ddots & & \\
\bar{w}_{k-1} & & & \bar{w}_{1} & 1 \\
0 & \ldots & & 0 & \bar{w}_{2^{s}-k+1}
\end{array}\right|
$$

Expanding this determinant along the bottom row yields

$$
\left(1, \ldots, 1,2^{s}-k+1\right)=\bar{w}_{2^{s}-k+1} \operatorname{det}\left|\begin{array}{cccc}
\bar{w}_{1} & 1 & & 0 \\
\bar{w}_{2} & \bar{w}_{1} & 1 & \\
\vdots & & \ddots & 1 \\
\bar{w}_{k-1} & & \cdots & \bar{w}_{1}
\end{array}\right|
$$


We can now rebuild this matrix into a $(k \times k)$ matrix by adding a 1 in the upper left-hand corner and appropriate terms down the first row and column. This is precisely the determinantal expansion for $w_{k-1}$. The result now follows.

Proposition 3.2. In $H^{*}\left(G_{k}\left(\mathbf{R}^{2^{+}+1}\right) ; \mathbf{Z}_{2}\right)$

$$
w_{1}^{2^{s}}=\bar{w}_{2^{s}+1-k} w_{k-1} \text {. }
$$

PRoof. We compute the image of $w_{1}^{2^{s}}+\bar{w}_{2^{3}+1-k} w_{k-1}$ under the injection

$$
\rho^{*}: H^{*}\left(G_{k}\left(\mathbf{R}^{2^{3}+1}\right) ; \mathbf{Z}_{2}\right) \rightarrow H^{*}\left(\operatorname{Flag}\left(\mathbf{R}^{2^{s+1}}\right) ; \mathbf{Z}_{2}\right) \text {. }
$$

Certainly, it suffices to show this image lies in the ideal $S_{2^{j}+1}$ generated by the elementary symmetric functions in the indeterminates $e_{1}, \ldots, e_{2^{s}+1}$. According to equation (2.1) we have

$$
\begin{aligned}
\rho^{*}\left(w_{1}^{2^{s}}+\bar{w}_{2^{s}+1-k} w_{k-1}\right) & \\
& =\sigma_{1}\left(e_{1}, \ldots, e_{k}\right)^{2^{s}}+\sigma_{k-1}\left(e_{1}, \ldots, e_{k}\right) \sigma_{2^{y+1}+k}\left(e_{k+1}, \ldots, e_{2^{y+1}}\right) \\
& =e_{1}^{2^{s}}+\cdots+e_{k}^{x^{s}}+\sum_{i=1}^{k}\left(e_{1} \cdots \hat{e}_{i} \cdots e_{k}\right) e_{k+1} \cdots e_{2^{y}+1}
\end{aligned}
$$

where ^denotes that the expression is omitted. Hence it suffices to show that for any $i<k$, the following polynomial lies in the ideal $S_{2^{\prime}+1}$,

$$
e_{i}^{2^{s}}+\prod_{j \neq i} e_{j} .
$$

As in Proposition 2.1 we recall the basic polynomial relation

$$
e_{i}^{2^{s}+1}-\sigma_{1} e_{i}^{2^{s}}+\cdots-e_{1} \cdots e_{2^{s+1}}=0 .
$$

We can now formally remove a factor of $e_{i}$ and get

$$
e_{i}^{2^{s}-\sigma_{1}} e_{i}^{2^{s}-1}+\cdots-\prod_{j \neq i} e_{j}=0 .
$$

Rearranging terms appropriately yields the desired expression.

THEOREM 3.3. If $k \geqslant 2$, then in $H^{*}\left(G_{k}\left(\mathbf{R}^{2^{s+1}}\right) ; \mathbf{Z}_{2}\right), w_{1}^{2^{2+1}-2} \neq 0$.

Proof. We proceed by induction on $k$. If $k=2$ then the result follows from Proposition 2.6. Inside $H^{*}\left(G_{k}\left(\mathbf{R}^{2^{3}+1}\right) ; \mathbf{Z}_{2}\right)$, we already know, by Propositions 3.1 and 3.2, that $w_{1}^{2^{2}} \neq 0$. But, it is clear that $w_{1}^{2^{3}+x} \neq 0$ if $w_{1}^{x} \neq 0$ inside $H^{*}\left(G_{k-1}\left(\mathbf{R}^{2^{3}-1}\right) ; \mathbf{Z}_{2}\right)$. By induction, we can assume that $w_{1}^{2^{*}-2} \neq 0$ in the cohomology of the space $G_{k-1}\left(\mathbf{R}^{2^{s-1}+1}\right)$. Hence, by Theorem 1.1(ii), $i^{*}\left(w_{1}\right)=w_{1}$ where $i$ is the inclusion

$$
i: G_{k-1}\left(\mathbf{R}^{2^{s-1}+1}\right) \rightarrow G_{k-1}\left(\mathbf{R}^{2-1}\right) \text {. }
$$

So we see that $w_{1}^{2^{3}-2} \neq 0$ in $H^{*}\left(G_{k-1}\left(\mathbf{R}^{2-1}\right) ; \mathbf{Z}_{2}\right)$. Now, by the remark above,

$$
w_{1}^{2^{2}+\left(2^{2}-2\right)}=w_{1}^{2^{s+1}-2} \neq 0
$$

in $H^{*}\left(G_{k}\left(\mathbf{R}^{2^{3}+1}\right) ; \mathbf{Z}_{2}\right)$ and the induction is complete.

Combining the results of $\$ \S 2$ and 3 we obtain the following estimate on the height of $w_{1}$. 
THEOREM 3.4. If $1<k \leqslant n$ then the height of $w_{1}$ in $H^{*}\left(G_{k}\left(\mathbf{R}^{n+k}\right) ; \mathbf{Z}_{2}\right)$ is $2^{\left\langle\log _{2}(n+k)\right\rangle}-\varepsilon$ where $\varepsilon$ is either 1 or 2 and $\langle x\rangle$ denotes the smallest integer $\rangle x$ (unless $k=2^{t}=n$ in which case we can only claim that $1<\varepsilon<2^{t}$ ).

Proof. By Corollary 2.5 , the height of $w_{1}$ is less than $2^{\left\langle\log _{2}(n+k)\right\rangle}$. It remains to show the height of $w_{1}$ is at least $2^{\left\langle\log _{2}(n+k)\right\rangle}-2$. By Theorem 3.3 it suffices to find an $s$ such that $2<2^{s}+1-k \leqslant n$. If we let $s=\left\langle\log _{2}(k+1)\right\rangle$ we obtain the minimal solution for the first inequality. Writing $k=2^{s}+i, 0<i<2^{s}$, we can compute

$$
2^{\left\langle\log _{2}(k+1)\right\rangle}+1-k=2^{s+1}+1-\left(2^{s}+i\right)=2^{s}+1-i .
$$

Now, the right-hand side of the above equation is $\langle k$ if and only if $i>1$. So if $k \neq 2^{t}$ then the result holds for all $n \geqslant k$. Similarly, if $k=2^{t}$ we only have the result for $n \geqslant k+1$. This completes the proof.

Modulo the ambiguity concerning $\varepsilon$, this theorem gives a very good picture of how the height of $w_{1}$ varies: If we fix our attention on subspaces of dimension $k \neq 2^{t}$ (respectively $k=2^{t}$ ), and let $n$ vary from $k$ (respectively $k+1$ ) to infinity, the heights of $w_{1}$ are nondecreasing. Within the intervals $\left[2^{s}-k+1,2^{s+1}-k\right]$ of length $2^{s}$, the height of $w_{1}$ is either $2^{s+1}-2$ or $2^{s+1}-1$, so there must be a cutoff point at which the height jumps up one. For $k=2$, it is clear from Proposition 6 of the Appendix that the cutoff point never comes, i.e. $\varepsilon=2$ always. Empirical computations on the MACSYMA symbolic manipulation system at MIT indicate that the cutoff point is solely a function of $k$ rather than the particular interval considered. We do not as yet have a prediction of the cutoff point in our intervals as a function of $k$, but we have been able to understand the cases $k=3,4$. The result is that for $k=3$ the cutoff is always one step down the interval and for $k=4$ it is at the very top, i.e. $\varepsilon=1$ always. We relegate the details to a future note.

4. Lusternik-Schnirelmann category. The Lusternik-Schnirelmann category [9] of a topological space is a fundamental numerical homotopy invariant. Indeed, from the analytic point of view, one knows that any smooth real-valued function on a compact, smooth manifold of category $n$ has at least $n$ critical points. T. Ganea, in Problem 1 of [7], inquires about the category of certain familiar manifolds and Lie groups. The case of the unitary groups $\mathrm{U}(n)$ and $\mathrm{SU}(n)$ have been handled by Singhof [13], and Schweitzer [12] has computed the category of $\operatorname{Sp}(2)$. It is not difficult to show that $\operatorname{cat}\left(G_{k}\left(\mathbf{C}^{n+k}\right)\right)=n k$. In this section, we obtain some lower bounds for the category of the real grassmanian $G_{k}\left(R^{n+k}\right)$ using our results on $w_{1}$ above. We begin by recalling the following

Definition 4.1. If a topological space $X=X_{0} \cup \cdots \cup X_{n}$, where each $X_{i}$, $0<i<n$, is a contractible open, then the Lusternik-Schnirelmann category of $X$ $(\operatorname{cat}(X))$ is at most $n$.

It is well known that $\operatorname{cat}(X) \leqslant \operatorname{dim}(X)$ and

Proposition 4.2 (EILENBERG [3]). If $x_{1}, \ldots, x_{n}$ are in $H^{*}(X)$ and the cup-product $x_{1} \cup \cdots \cup x_{n}$ is nonzero, then $\operatorname{cat}(X) \geqslant n$.

In particular, $\operatorname{cat}(X) \geqslant \operatorname{cup}(X)$ as defined in $\S 2$. It is now easy to obtain 
THEOREM 4.3. If $1<k \leqslant n$ then

$$
\operatorname{cat}\left(G_{k}\left(\mathbf{R}^{n+k}\right)\right) \geqslant 2^{\left\langle\log _{2}(n+k)\right\rangle}-2 \text {. }
$$

Proof. This follows immediately from Theorem 3.3 and Proposition 4.2.

In the Appendix, for the case $k=2$, we do slightly more work involving $w_{2}$ and get

Proposition 4.4. If $n=\left(2^{s}-1\right)+d, d<2^{s}$, then

$$
\operatorname{cat}\left(G_{2}\left(\mathbf{R}^{n+2}\right)\right) \geqslant 2\left(2^{s}-1\right)+d \text {. }
$$

In this section, we attempt a similar refinement for the case $k=3$. We recall a formula of Oproiu [10].

LEMMA 4.5. The following identity holds in $H^{*}\left(G_{3}\left(R^{n+3}\right) ; Z_{2}\right)$,

$$
w_{1}^{j}=\sum_{p, q}\left[\left(\begin{array}{c}
p+q-1 \\
q
\end{array}\right)\left(\begin{array}{c}
j \\
p+q-2
\end{array}\right)+\left(\begin{array}{c}
p+q+1 \\
q+1
\end{array}\right)\left(\begin{array}{c}
j+1 \\
p+q
\end{array}\right)\right](p, q, j-p-q) .
$$

This lemma follows by a fairly straightforward induction and has been used by Oproiu [10] to look at embedding questions. We will use it to find a good expression for the fundamental class as a monomial in $w_{1}$ and $w_{2}$. It is now possible to show

LEMMA 4.6. The following identity holds in $H^{*}\left(G_{3}\left(\mathbf{R}^{2^{2}+1}\right) ; \mathbf{Z}_{2}\right)$,

$$
w_{1}^{2^{s+1}-2}=\sum_{i=2}^{2^{s-1}}\left(i, 2^{s}-i, 2^{s}-2\right) \text { and } w_{1}^{2^{s+1}-1}=0 .
$$

Proof. From Proposition 3.1 we get $w_{1}^{2^{s}}=\left(1,1,2^{s}-2\right)$. The last entry in this Schubert cycle is the codimension. It is clear from the Pieri formula that this property is inherited by any cycle appearing in higher powers of $w_{1}$. Therefore, the summands mentioned are the only ones that can appear. That they do appear follows from the easy computation

$$
\left(\begin{array}{c}
2^{s}-1 \\
2^{s}-i
\end{array}\right)\left(\begin{array}{c}
2^{s+1}-2 \\
2^{s}-2
\end{array}\right)+\left(\begin{array}{c}
2^{s}+1 \\
2^{s}-i+1
\end{array}\right)\left(\begin{array}{c}
2^{s+1}-1 \\
2^{s}
\end{array}\right) \equiv 1(\bmod 2)
$$

since the third binomial coefficient is zero and the first two are always one. To see that the next power of $w_{1}$ vanishes, we observe that for $3<i<2^{s-1}-1$,

$$
\left(i, 2^{s}-i, 2^{s}-2\right) w_{1}=\left(i+1,2^{s}-i, 2^{s}-2\right)+\left(i, 2^{s}-i+1,2^{s}-2\right)
$$

and if $i=2$ (respectively $i=2^{s-1}$ ) one omits the second (respectively first) term. It is then easy to observe that every cycle appears twice.

We now consider the effect of multiplying by $w_{2}$. For the moment, we define

$$
F(k)=\sum_{i=2+2 k}^{k+2^{s-1}}\left(i, 2^{s}-i+2 k, 2^{s}-2\right)
$$

LEMMA 4.7. The following identity holds in $H^{*}\left(G_{3}\left(\mathbf{R}^{2^{s}+1}\right) ; \mathbf{Z}_{2}\right), \quad F(k+1)=$ $F(k) w_{2}$. 
Proof. We assume $k=0$ since the general case is identical. Observe that $w_{2}=\bar{w}_{1}^{2}+\bar{w}_{2}$. It follows from the Pieri formula that $\left(a_{1}, a_{2}, a_{3}\right) w_{2}=\Sigma\left(b_{1}, b_{2}, b_{3}\right)$ where the sum ranges over $\left(b_{1}, b_{2}, b_{3}\right)$ such that $a_{i}<b_{i}<\min \left(a_{i+1}, a_{i}+1\right)$ and $b_{1}+b_{2}+b_{3}=2+a_{1}+a_{2}+a_{3}$. It is then easy to check that for $i>2$,

$$
\left(i, 2^{s}-i, 2^{s}-2\right) w_{2}=\left(i+1,2^{s}-i+1,2^{s}-2\right)
$$

and when $i=2$ we get zero. The result now follows.

Finally, we come to the desired expression for the fundamental class.

CoROllaRY 4.8. The following identity holds in $H^{*}\left(G_{3}\left(\mathbf{R}^{2^{s+1}}\right) ; \mathbf{Z}_{2}\right)$,

$$
w_{1}^{2^{s+1}-2} w_{2}^{2^{s-1}-2}=\left(2^{s}-2,2^{s}-2,2^{s}-2\right) .
$$

Proof. Using Lemma 4.7 we get

$$
w_{1}^{2^{s+1}-2} w_{2}^{2^{s-1}-2}=F\left(2^{s-1}-2\right)=\left(2^{s}-2,2^{s}-2,2^{s}-2\right) .
$$

THEOREM 4.9. The cup-length of $G_{3}\left(\mathbf{R}^{2^{s+1}}\right)$ is $2^{s+1}+2^{s-1}-4$.

Proof. By Lemma 4.6 we cannot increase the contribution of $w_{1}$ to the monomial. But then any other way of writing a monomial of high graded degree will decrease the ungraded degree. So our monomial is the best possible.

We now summarize the improved lower bound on the Lusternik-Schnirelmann category for $k=3$ that our computations yield.

THEOREM 4.10. If [ ] denotes the integral part function then

$$
\frac{5}{2}\left(2^{\left[\log _{2}(n+2)\right]}-2\right)+1<\operatorname{cat}\left(G_{3}\left(\mathbf{R}^{n+3}\right)\right)<3 n .
$$

In particular, if $n=2^{s}-2$, then $\operatorname{cat}\left(G_{3}\left(\mathbf{R}^{n+3}\right)\right)>\frac{5}{2} n-1$.

Proof. Choose the largest possible $s$ such that $2^{s}-2<n$, i.e. $s=\left[\log _{2}(n+2)\right]$. Then we have the inclusion $G_{3}\left(\mathbf{R}^{2^{s}+1}\right) \rightarrow G_{3}\left(\mathbf{R}^{n+3}\right)$ and can consider the induced map on cohomology. Since Whitney classes are mapped to themselves we see that the monomial of Corollary 4.8 in the cohomology of $G_{3}\left(R^{n+3}\right)$ is mapped to a nonzero element; $a$ fortiori, it is nonzero. The result now follows.

An additional piece of information that is available on the question of category is

THEOREM 4.11 (BERSTEIN-SCHWARTZ [2]). If $X$ is a closed, connected n-manifold with $\pi_{1}(X) \approx \mathbf{Z}_{2}$, then $\operatorname{cat}(X)=\operatorname{dim}(X)$ iff $w^{\operatorname{dim}(x)} \neq 0$, where $w$ is the nonzero element of $H^{1}\left(X ; Z_{2}\right)$.

This result allows us to assert the strict inequality cat $<\operatorname{dim}$ in all but the exceptional cases for the spaces $G_{k}\left(R^{n+k}\right)$. In particular, we can compute $\operatorname{cat}\left(G_{2}\left(\mathbf{R}^{2^{s}+2}\right)\right)=2^{s+1}-1$. It would be interesting now to find some significant upper bounds on category. A possible approach might be to look for smooth real-valued functions on $G_{k}\left(\mathbf{R}^{n+k}\right)$ with a small number of critical points. Of course, our results give lower bounds on the number of critical points of such functions. 
Appendix. Eliminating the normal classes. In this Appendix, we provide an algebraic structure theorem that works over any field $F$. Since this work is purely algebraic, independent of the topological context, we suppress any mention of cohomology and work directly with the relevant algebra.

THEOREM 1. Let $L(k, n)$ denote the graded F-algebra $F\left[w_{1}, \ldots, w_{k}, z_{1}, \ldots, z_{n}\right] / I(k, n)$ where $\operatorname{dim}\left(w_{i}\right)=i=\operatorname{dim}\left(z_{i}\right)$ and $I(k, n)$ is generated by the single nonhomogeneous relation

$$
\left(1+w_{1}+\cdots+w_{k}\right)\left(1+z_{1}+\cdots+z_{n}\right)-1=0 .
$$

Then $L(k, n) \approx F\left[w_{1}, \ldots, w_{k}\right] / J(k, n)$ where $J(k, n)$ is generated by the $k$ homogeneous relations $f_{1, n}, \ldots, f_{k, n}$ given recursively by

$$
\left(\begin{array}{c}
f_{1, n} \\
f_{2, n} \\
\vdots \\
f_{k, n}
\end{array}\right)=\left(\begin{array}{ccccc}
w_{1} & 1 & & & 0 \\
w_{2} & 0 & 1 & \cdots & 0 \\
\vdots & & & \ddots & \\
w_{k} & 0 & \cdots & & 0
\end{array}\right)^{n+1}\left(\begin{array}{c}
1 \\
0 \\
\vdots \\
0
\end{array}\right) .
$$

Before giving the proof of this result we record the simple facts on binomial coefficients that we will require.

LEMMA 2. (i) $\left({ }^{2}-1-i\right) \equiv 0(\bmod 2)$ if $i>0$.

(ii) $\left(2^{3}-2-i\right) \equiv 0(\bmod 2)$ if $i \equiv 0(\bmod 2)$ and $i>0$.

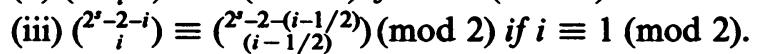

(iv)

$$
\begin{array}{r}
\frac{\left(i_{1}+\cdots+i_{k}\right) !}{\left(i_{1} !\right) \cdots\left(i_{k} !\right)}=\left(i_{1}+\cdots+i_{k}-1\right) !\left(\left(\frac{1}{\left(i_{1}-1\right) ! \cdots\left(i_{k} !\right)}\right)+\cdots\right. \\
\left.+\left(\frac{1}{\left(i_{1} !\right) \cdots\left(i_{k}-1 !\right)}\right)\right)
\end{array}
$$

Proof. (i)-(iii) are an application of Lemma 2.6 of [15, p. 5] and (iv) is immediate.

We now have the

Proof of Theorem 1. Since (*) is symmetric in $w$ and $z$ we can assume without any loss of generality that $k<n$. We proceed by induction on $n$. The case $n=0$ is trivial. In the general situation, we first observe that the single nonhomogeneous relation (*) encodes $(n+k)$ homogeneous relations. The first $n$ of these merely express the $z$ 's as polynomials in the $w$ 's. The remaining $k$ relations (after substitution) give generators for the contraction of the ideal $I(k, n)$ back to the $k$-variable polynomial ring $F\left[w_{1}, \ldots, w_{k}\right]$. Specifically, we have

$$
z_{m}=\sum \frac{\left(i_{1}+\cdots+i_{k}\right) !}{\left(i_{1}\right) ! \cdots\left(i_{k}\right) !} w_{1}^{i} 1 \cdots w_{k}^{i} k
$$


where $1<m<n$ and the sum is over all $k$-tuples $\left(i_{1}, \ldots, i_{k}\right)$ satisfying $i_{1}+2 i_{2}+$ $3 i_{3}+\cdots+k i_{k}=m$. The nontrivial relations then become

$$
f_{j, n}:=w_{j} z_{n}+w_{j+1} z_{n-1}+\cdots+w_{k} z_{n-k+j}=0
$$

where $1<j<k$. We now claim $f_{1, n}=z_{n+1}$, i.e. $z_{n+1}=w_{1} z_{n}+\cdots+w_{k} z_{n-k+1}$.

But this amounts precisely to the identity of multinomial coefficients contained in Lemma 2(iv). It now suffices to show $f_{j, n+1}=w_{j} f_{1, n}+f_{j+1, n}$ where $1<j<k$ and by convention $f_{k+1, n}=0$. We compute

$$
\begin{aligned}
w_{j} f_{1, n}+f_{j+1, n} & =w_{j} z_{n+1}+f_{j+1, n} \\
& =w_{j} z_{n+1}+w_{j+1} z_{n}+\cdots+w_{k} z_{n-k+j+1}=f_{j, n+1} .
\end{aligned}
$$

This completes the proof.

In practice, Theorem 1 can be very useful in deciding the triviality of polynomials in the $w_{i}$ 's. A somewhat pleasant recursive formula is

CoROllary 3. $f_{1, n}=\sum_{j=1}^{k} w_{j} f_{1, n-j}$.

Proof. Iterate the recursive expression for $f_{1, n}$ contained in Theorem 1.

For the rest of this section we will assume that $F=Z_{2}$ and $k=2$. We will write $f_{n}$ for $f_{1, n}$ and $g_{n}$ for $f_{2, n}$. The first step in our task of showing that high powers of $w_{1}$ survive is

Proposition 4. The element $w_{2}^{n}$ is not in the ideal $J(2, n)$, i.e. $w_{2}^{n}$ is nonzero in the $\mathbf{Z}_{2}$-algebra $L(2, n)$.

Proof. We proceed by induction on $n$, the case $n=0$ being trivial. Suppose there exist polynomials $c, d$ such that $w_{2}^{n+1}=c f_{n+1}+d g_{n+1}$. Since $g_{n+1}=w_{2} f_{n}, w_{2}$ divides into $c f_{n+1}$. But $f_{n+1}$ contains the monomial $w_{1}^{n+2}$, so $w_{2}$ divides into $c$. Then $w_{2}^{n}=\left(c / w_{2}\right) f_{n+1}+d f_{n}=\left(c / w_{2}\right)\left(w_{1} f_{n}+g_{n}\right)+d f_{n}=\left(c w_{1} / w_{2}+d\right) f_{n}+\left(c / w_{2}\right) g_{n}$. This completes the induction step and the proof.

REMARK. This proposition also follows immediately from Lemma 1.2 using the Schubert calculus, but we prefer this more elementary algebraic approach.

Proposition 5. If $n=\left(2^{s}-1\right)+d, d<2^{s}$, then $w_{2}^{n}+w_{1}^{2\left(2^{s}-1\right)} w_{2}^{d}=0$ inside $L(2, n)$. Thus $w_{1}^{2\left(2^{s}-1\right)} w_{2}^{d}$ is nonzero.

Proof. We induct on $d$. If $d=0$, the assertion is $w_{2}^{n}+w_{1}^{2 n}=z_{n-1} f_{n}$ where $n=2^{s}-1$. First we claim $z_{n}=w_{1}^{n}$, for such $n$. This follows from Lemma 2(i). Hence the right-hand side of the above equation becomes

$$
z_{n-1} f(n)=z_{n-1}\left(w_{1} z_{n}+w_{2} z_{n-1}\right)=w_{1}^{n+1} z_{n-1}+w_{2} z_{n-1}^{2}
$$

where

$$
z_{n-1}=\sum_{i=0}^{2^{s-1}-1}\left(\begin{array}{c}
2^{s}-2-i \\
i
\end{array}\right) w_{1}^{2 s-2 i-2} w_{2}^{i} .
$$

Letting $i=0$ in $z_{n-1}$, the first monomial of (*) gives $w_{1}^{n+1} w_{1}^{n-1}=w_{1}^{2 n}$, and letting $i=2^{s-1}-1$ in $z_{n-1}$, the second monomial of $(*)$ contributes $w_{2}\left(w_{2}^{2^{s-1}-1}\right)^{2}=$ $w_{2}^{n}$. We must check that everything else cancels. If $y$ is not 0 or $n$ then the 
coefficient of $w_{1}^{2 n-2 y} w_{2}^{y}$ is

$$
\begin{gathered}
\left(\begin{array}{c}
n-y-1 \\
y
\end{array}\right)+\left(\begin{array}{c}
n-(y-1 / 2)-1 \\
(y-1 / 2)
\end{array}\right) \quad \text { if } y \equiv 1(\bmod 2) \\
\left(\begin{array}{c}
n-y-1 \\
y
\end{array}\right) \text { if } y \equiv 0(\bmod 2) .
\end{gathered}
$$

Lemma 3.2(ii), (iii) verifies that these coefficients vanish. For the induction step, suppose

$$
w_{2}^{n}+w_{1}^{2\left(2^{s}-1\right)} w_{2}^{d}=c f_{n}+d g_{n}
$$

where $n=\left(2^{s}-1\right)+d, d<2^{s}-1$. Multiplying by $w_{2}$, we obtain

$$
\begin{aligned}
w_{2}^{n+1}+w_{1}^{2(2-1)} w_{2}^{d+1} & =c w_{2} f_{n}+d w_{2} g_{n} \\
& =c g_{n+1}+d w_{2}\left(f_{n+1}+w_{1} f_{n}\right) \\
& =d w_{2} f_{n+1}+\left(c+d w_{1}\right) g_{n+1} .
\end{aligned}
$$

Indeed, we can see that the new coefficients $c^{\prime}, d^{\prime}$ are given by

$$
\left(\begin{array}{l}
c^{\prime} \\
d^{\prime}
\end{array}\right)=\left(\begin{array}{ll}
0 & w_{2} \\
1 & w_{1}
\end{array}\right)\left(\begin{array}{l}
c \\
d
\end{array}\right)
$$

This completes the proof.

Remark. Of course, Proposition 5 immediately implies Proposition 2.6, since $H^{*}\left(G_{2}\left(\mathbf{R}^{n+2}\right) ; \mathbf{Z}_{2}\right) \approx L(2, n)$.

The above proposition indicates that the height of $w_{1}$ is at least $2\left(2^{s}-1\right)$. By the results of $\$ 2$ we know that $w_{1}^{2^{2+1}}=0$. The ambiguity is resolved by the following computations.

Proposition 6. $w_{1}^{2^{s+1}-1}=0$ in $L(2, n)$, where $n=\left(2^{s}-1\right)+d, d<2^{s}$.

Proof. It suffices to show this holds in $L(2, r)$ where $r=2^{s+1}-2$. In that case, we have relation $f_{r} w_{1} z_{r}+w_{2} z_{r-1}=0$ in $L(2, r)$. Separating the first monomial to the left, one gets

$$
w_{1}^{r+1}=\sum_{i=1}^{r / 2}\left(\begin{array}{c}
r-i \\
i
\end{array}\right) w_{1}^{r-2 i+1} w_{2}^{i}+\sum_{j=0}^{(r-2) / 2}\left(\begin{array}{c}
r-j-1 \\
i
\end{array}\right) w_{1}^{r-2 j-1} w_{2}^{j+1}
$$

If $y>0$ then the coefficient of $w_{1}^{r+1-2 y} w_{2}^{y}$ is

$$
\left(\begin{array}{c}
r-y \\
y
\end{array}\right)+\left(\begin{array}{c}
r-y \\
y-1
\end{array}\right)=\left(\begin{array}{c}
r+1-y \\
y
\end{array}\right)
$$

But again, Lemma 2(ii) shows this binomial coefficient disappears.

We record a final consequence of these results which ties together Propositions 5 and 6.

COROllary 7. $w_{1}^{2 n}$ is not in $J(2, n)$ if and only if $n=2^{s}-1$, for some s. 


\section{REFERENCES}

1. I. N. Berstein, I. M. Gelfand and S. I. Gelfand, Schubert cells and cohomology of the spaces $G / P$, Russian Math. Surveys (1973), 1-26.

2. I. Berstein, On the Lusternik-Schnirelmann category of grassmanians, Proc. Cambridge Philos. Soc. 79 (1976), 129-134.

3. I. Berstein and T. Ganea, The category of a map and of a cohomology class, Fund. Math. 50 (1961/62), 265-279.

4. A. Borel, Sur la cohomologie des espaces fibrés principaux et des espaces homogènes de groupes de Lie compacts, Ann. of Math. 57 (1953), 115-207.

5. S. Chern, On the multiplication in the characteristic ring of a sphere bundle, Ann. of Math. 49 (1948), 362-372.

6. C. Ehresmann, Sur la topologie de certains espaces homogines, Ann. of Math. 35 (1934), 396-443.

7. T. Ganea, Some problems on numerical homotopy invariants, Symposium on Algebraic Topology, Lecture Notes in Math., vol. 249, Springer-Verlag, Berlin, 1971, pp. 23-30.

8. F. Hirzebruch, Topological methods in algebraic geometry, Springer-Verlag, New York, 1966.

9. L. Lusternik and L. Schnirelmann, Methodes topologiques dans les problemes variationnels, Hermann, Paris, 1934.

10. V. Oproiu, Some non-embedding theorems for the grassman manifolds $G_{2, n}$ and $G_{3, n}$, Proc. Edinburgh Math. Soc. 20 (1977), 177-185.

11. H. Schubert, Kalkül der abzählenden Geometrie, Teubner, Leipzig, 1879.

12. P. Schweitzer, Secondary cohomology operations induced by the diagonal mapping, Topology 3 (1965), 337-355.

13. W. Singhof, On the Lusternik-Schnirelmann category of Lie groups. I, II, Math. Z. 145 (1975), 111-116; 151 (1976), 143-148.

14. R. Stanley, On some combinatorial aspects of the Schubert calculus, Combinatoire et représentation du groupe symétrique, Strasbourg, 1976.

15. N. Steenrod and D. Epstein, Cohomology operations, Ann. of Math. Studies, Princeton Univ. Press, Princeton, N. J., 1962.

Department of Mathematics, Massachusetts Institute of Technology, Cambridge, MASSACHUSETTS 02139

Mathematical InStTtute, OXFord UnIVERSTTY, OXFord, ENGLAND

Current address: Department of Mathematics, Yale University, New Haven, Connecticut 06520 